\title{
Orbital Spin: A New Hypothesis to Explain Precession of Equinox-The Third Motion of Earth
}

\author{
Rama Chandra Murthy Mothe* \\ Greater Hyderabad Municipal Corporation, Hyderabad, India \\ Email: ramachandramurthymothe@gmail.com
}

Received 1 November 2013; revised 1 December 2013; accepted 10 December 2013

Copyright (c) 2014 by author and Scientific Research Publishing Inc.

This work is licensed under the Creative Commons Attribution International License (CC BY). http://creativecommons.org/licenses/by/4.0/

(c) (i) Open Access

\begin{abstract}
In this paper, the phenomena of Earth's motion about its own axis, the ecliptic plane of the Earth's orbit around the Sun, the definitions of equinoxes, the precession of equinoxes, Earth's wobble and other astronomical terminology are briefly described. Some of the existing theories explaining the precession of equinox and their inadequacies are brought out. New Hypothesis is that precession of equinoxes is a direct result of Orbital spin of Earth in a retrograde direction-a celestial phenomenon similar to that of Moon's Orbital spin around the Earth. The study of Moon's orbit round the Earth reveals the exact movement of Earth's orbit, which causes precession of equinoxes without any ambiguity. The analogy presented herein demonstrates the plausible hypothesis.
\end{abstract}

\section{Keywords}

Precession of Equinox; Third Motion of Earth; Orbital Spin

\section{Introduction}

The Vernal equinox point emerges when Earth completes an orbit around the Sun annually. It is the point of intersection of Earth's orbit with equatorial plane when Earth crosses the equatorial plane (from Southern hemisphere in to Northern hemisphere). The fundamental reference point is the Vernal equinox from which longitudes of planets measured along the ecliptic [1]. The uniform backward movement of Vernal equinox along the ecliptic is the precession of the equinox [1]. The precession of equinox also refers to emergence of Vernal equinox on 20th of March at an advanced point ahead of its scheduled emergence point on the equatorial plane at the rate of 50.3" per annum. The motion of Earth's axis (North Pole) describes a circle of precession once in approximately

\footnotetext{
*Retired Executive Engineer.
} 
25772 years at an annual rate of 50.3" arc in a retrograde motion in the overhead sky about star Polaris [2]. Nicolas Copernicus referred to the precession of equinox (Poland 1473 AD) as "Third motion of the Earth" [3].

The cause of apparent motion of precession of equinoxes attributed presumptuously to movement of Earth's axis. Sir Isaac Newton (1678 AD) explained movement of Earth's axis as a direct consequence of Earth's wobble, which is due to the gravitational pull on Earth's equatorial bulge by Moon and Sun [4]. This North Pole movement in turn is supposed to be the cause of precession of equinox. Scientists later on deferred with this version and made alternative propositions. However, no definite movement of Earth is identified which could possibly explain comprehensively the cause of precession of equinoxes, which remained unexplained as on today.

The equinoxes moved westward along the ecliptic relative to the fixed stars opposite to the motion of the Sun along ecliptic. The precession of Earth's called precession of the equinoxes in early days. Later on as early as 1863, it is general precession, instead of precession of equinoxes based on detailed classification determined by mathematical derivates of gravitational forces between planets [5].

The classification was due to new hypothesis that ecliptic itself moved slightly because of gravitational pull of planets which was named planetary precession, the predominant component being Lunisolar precession. The combination is termed as general precession.

The new hypothesis postulated the theory that Lunisolar precession is caused by the gravitational forces of the Moon and Sun on equatorial bulge which in turn causes Earth's axis to move with respect to inertial space. Lunisolar precession being 500 times intense than all other planets is put together.

The International Astronomical Union in 2006 was recommended to name lunisolar precession (major component) as the precession of equator and precession due to other planets (minor component) as the precession of the ecliptic, the combination being general precession.

The celestial equator is the plane of the Earth's equator extended to the celestial sphere. The plane of the ecliptic is the plane of the Earth's orbit. Imagine the intersection of the ecliptic plane with the celestial equator at two points, which occur on Vernal equinox day (20th March) and Autumnal equinox day (September 23) [6]. The Sun is South of the equator for half the year and North of it for the other half (Figure 1).

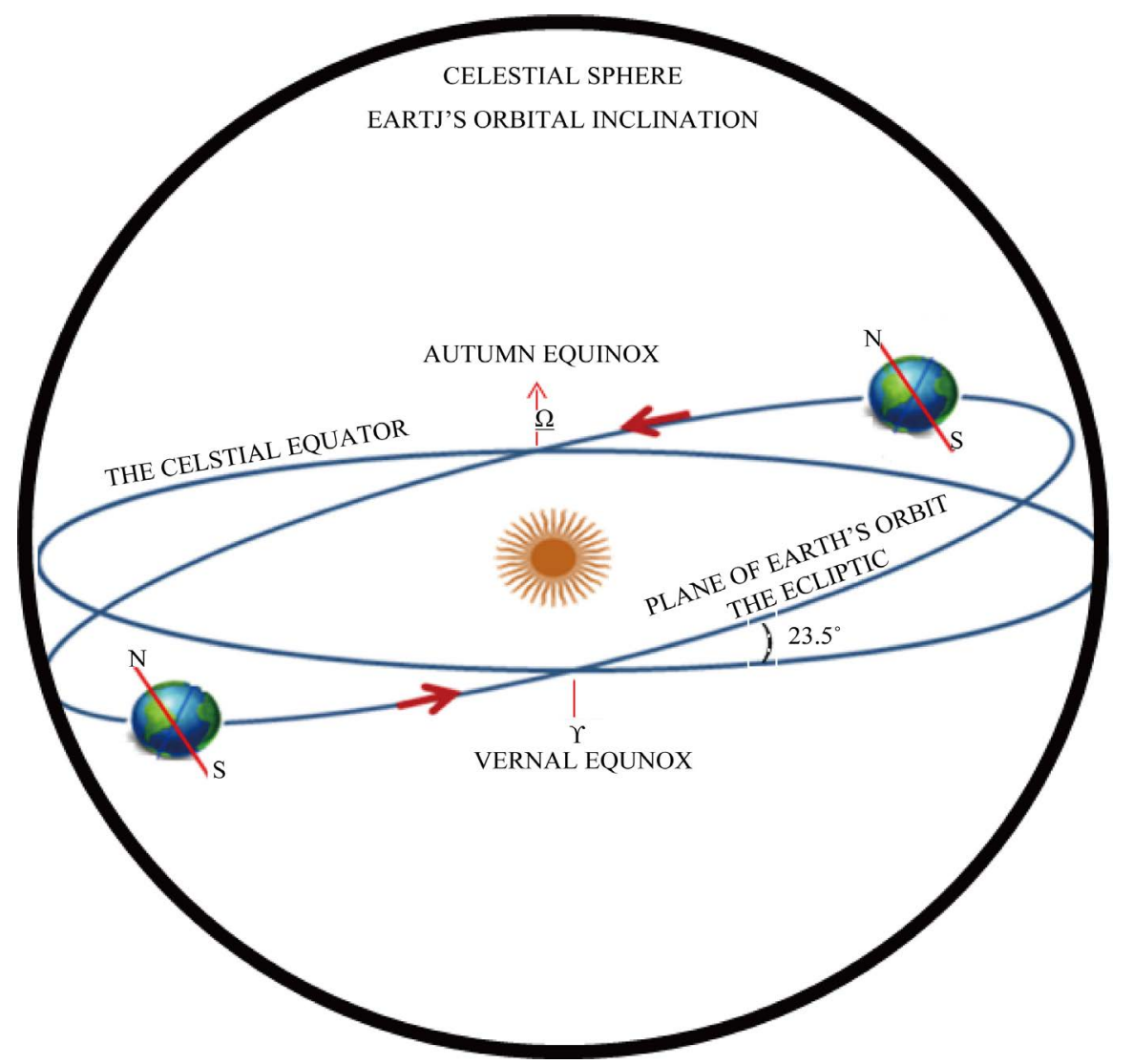

Figure 1. Vernal and autumnal equinoxes-Ascending and descending nodes of Earth. 
When the Earth crosses the plane of the celestial equator twice each year, the length of the day and night is equal (Equinox) and the Earth's axis does not tilt towards or away from the Sun [7]. The Vernal equinox is the "zero" point of the zodiac and the longitudes of all the planets are reckoned from this as initial point i.e.; "zero" degrees of sign Aries. Similarly, the Autumnal equinox, which is 180 degrees away and diametrically opposite to "zero" Degrees of Aries, is “zero" degrees in the Sign Libra of the Zodiac. Thus Ascending node of the Earth is Vernal equinox and the Descending node of the Earth is Autumnal equinox. In fact, all the planets in solar system orbit round the Sun in a similar mode [8]. The nodes of Moon (Rahu and Ketu in Indian astrology are Dragon's head and tail respectively in Western astrology) are discussed herein to extend the analogy to precession of equinoxes [9].

Earth's transit around the sun once in 365.256 solar days is an elliptical orbit, which is fixed [10]. It means that the points of intersection (equinoxes) on equatorial plane generated by Earth's orbital plane should have been rigid and fixed. However, they are not fixed and show precession.

On observation, astronomers of yesteryears found that the Vernal equinox ("zero" degrees of zodiac) is shifting by 50.3 seconds arc (modern value) annually in retrograde motion in the zodiac because of which the longitudes of planets are found to be increased in equal amount. The annual retrograde shift of Vernal equinox by 50.3 seconds arc is termed as Precession of equinox. The amount of shift in longitudes is the difference observed between two successive points of intersection generated by Earth's orbital plane on Equatorial plane. The orbital period elapsed between two successive points being "one” solar year or approximately 365.256 solar days [11].

The backward movement of Vernal equinox annually at the rate of 50.3" in the zodiac is the precession of equinoxes and named as "Third motion of Earth".

The precession causes the cycle of season (tropical year) to be about 20.4 Minutes less than the time for the earth to return to the same position with respect to the stars. This results in a slow change (1 day every 71 calendar years) in the position of the sun with respect to the stars at the equinox.

So while Polaris is currently our North Star it was not always so. The Pole star was Thuban in 3000 BC and Vega will be in 14,000 AD (Figure 2) [12]. Therefore, we are off center. The exact center of the sky is the Pole of the Ecliptic, directly above the Sun and Planets. The Center is in the middle of the Constellation Draco, the Dragon, and is the Pole of the Ecliptic.

Currently this annual motion is about 50.3 seconds of arc per year or 1 degree every 71.6 years. The process is

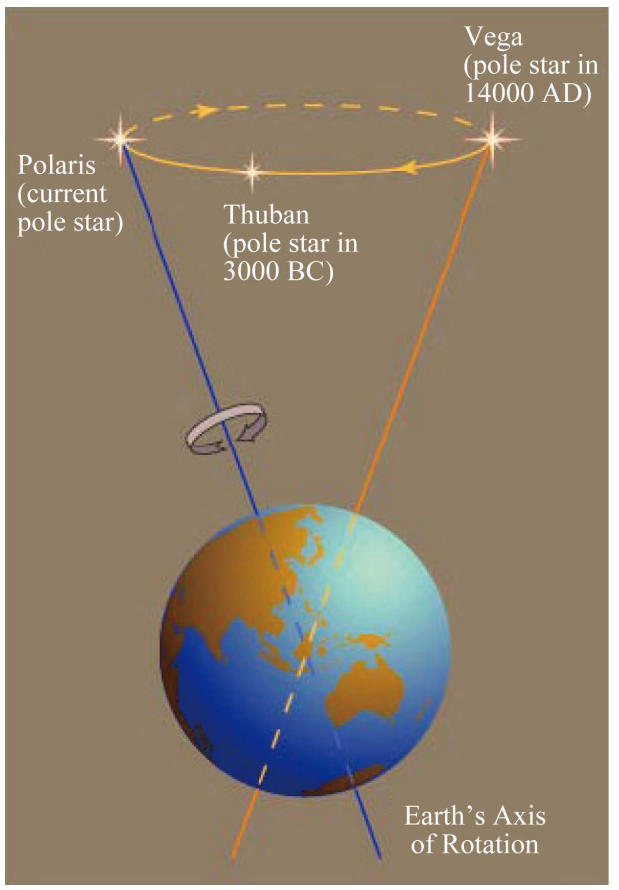

Figure 2. Circle of precession of North Pole. Credit: University of Hong Kong, department of physics. 
slow but cumulative. A complete precession cycle covers a period approximately 25,772 years in the platonic year during which time the equinox regresses a full 360 degrees through all constellations of the zodiac [2].

In Section 2, the history and some of the existing theories on Precession of Equinox are briefly described. In Section 3, the precession of equinoxes, as per the New hypothesis is due to direct result of Orbital spin of Earth in a retrograde direction-a celestial phenomenon similar to that of Moon's orbital spin around the Earth. The study of Moon's orbit round the Earth reveals the exact movement of Earth's orbit, which causes precession of equinoxes without any ambiguity. This analogy is described.

\section{Precession of Equinox-History and Existing Theories}

Earth orbits round the Sun in its ecliptic. The position of planets and Sun are determined with reference to the stars in the zodiac of different constellations distributed within a bandwidth of 8 degrees above (North) and below (South) of the ecliptic. The groups of stars in different constellation of the zodiac distributed over 12 signs, each sign constituting 30-degree measure [13].

The name of a bright star among the group of stars in a constellation is the name of the constellation [14]. The beginning star in the constellation is the starting point of sign in the zodiac. In Indian Astrology, the star Ashwin that is $\beta$ Arietis in Aries sign is starting point in the Zodiac, it is nearly 180 degrees opposite to the star Chitta that star Spica16 in Libra sign. The points of Vernal equinox and Autumnal equinox are the positions in the above stars of the zodiac. In Western Astrology also, Vernal equinox is the first point in $\beta$ Arietis in Aries sign [15].

Indian Astrologers studied the movement of stars continuously for long periods and noticed that the longitude of all the stars were increasing when reckoned from first point in Aries. In olden times, the observation was that the longitudes of all the planets increased by same amount every year. They observed that while the latitudes were constant the longitudes only were increased. They concluded that the ecliptic is not moving backwards but the vernal equinox is moving backwards and there by the longitude of all the stars were increasing [16].

N. C. Lahiri reformer of national calendar of India assumed the coincidence of sidereal ecliptic longitude with respect to stars with tropical ecliptic longitude with respect to Vernal equinox point in the year 285 AD in star Arietis in Zodiacal sign of Aries. Thus, the tropical longitude of Vernal equinox is assumed to be behind by 23.85 degrees from sidereal longitude that is fixed as of 2000 and is presently in the constellation of Pegasi in zodiacal sign of Pisces [16].

Hipparchus (127 BC) a Greek astronomer discovered that the positions of the equinoxes move westward along the ecliptic compared to the fixed star on the celestial sphere that is Zodiac.

The values of sidereal and tropical year were stated by Ptolemy's almagest where in precession is explained as the rotation of celestial sphere around a static geocentric Earth.

Both, Hipparchus and Ptolemy thought of precession in geocentric model [2]. Nicolaus Copernicus adduced the first hypothesis on precession as a direct consequence of a motion of the Earth's axis. He called the precession the third motion of the earth. Sir Isaac Newton (1687 AD) subsequently tried to explain precession of equinoxes because of gravitational pulls and pushes on earth [17]. However, scientists rejected Newton's hypothesis later on.

Translation of Surya sidhanta-ancient Indian Astronomical Treatise (Dated 490AD) as translated by Ebenezer Burgess estimates the precession of equinoxes at the rate of 54" of arc per year [16].

Subsequently an alternate hypothesis was developed to explain the precession of equinoxes. It states that from certain epoch the solstices have a motion of 8 degrees in the order of the signs after which they go back the same amount instead of proceeding through the entire sequence of Zodiac. It has thus a back and forth trepidation over an arc of 8 degrees.

One of the existing theories hypothesizes this third motion of Earth to be a direct result of Earth's wobble. The imaginary Earth's wobble is supposedly due to the differential gravitational forces of the Sun and Moon on the equatorial bulge of the earth. The hypothesis is differential gravitational force is causing oscillation of the earth or wobble of the earth on its axis duly shifting the position of North Pole with reference to star Polaris at an equal rate of 50.3 seconds in overhead sky, which is in turn causing precession of equinoxes on equatorial plane (Figure 3).

There are no associated terrestrial changes observed because of the gravitational disturbances caused by Sun and Moon at the equatorial bulge of the Earth to adduce the imaginary Earths' wobble as real. 


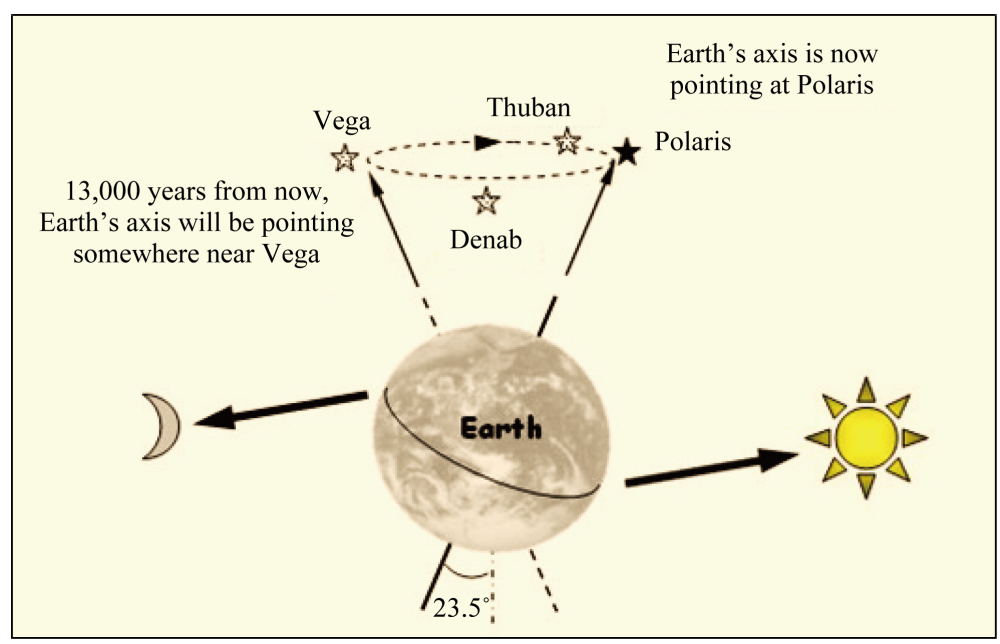

Figure 3. Imaginary Wobble of Earth. Credit: MyDarSky.org.

The Moon's gravitational pull on Earth's equator causes tidal waves in the oceans. When a similar presumption that Earth's wobble is due to gravitational pull and pushes on equatorial bulge is due to Moon and Sun, it is conspicuous that there are no associated disturbances on Earth's surface. Hence, the hypothesis of Earth's wobble is not credible.

When Earth spins on its axis in West to East direction (Anti clockwise) it is natural that North Pole of the axis moves in the same direction. It is how North Pole can describe a circle of precession about star Polaris in a clockwise direction opposite to the natural rotation of North Pole of the axis conspicuously remains unexplained. The hypothesis of Earth's wobble does not explain above contradiction.

Hence, the hypothetical proposition that the retrograde motion of North Pole is due to Earth's wobble is not credible. Whereas the circle of precision generated by North Pole in 25772 years in clockwise direction that is opposite to the natural direction of Earth's axis, as observed, however, indeed a fact. This phenomenon is due to realistic orbital movement of the Earth to which we shall refer to here after.

Another explanation to the Precession is observable in solar system motion. That it is not the Earth that wobbles but rather the motion of the solar system curving through space slowly changing the orientation of the solar system in relation to the fixed stars that produces the observable known as the precession of the equinox.

Another hypothesis is Luni-Solar precession. It believes that other planets in the solar system cause the ecliptic to rotate around an axis slowly whose longitude is 174 degrees measured on instantaneous ecliptic. The planetary precession indicates a shift of 0.47 seconds annually on the instantaneous equator. The sum of these two precessions results in precession of equinoxes.

All above explanations in the context conclusively failed to demonstrate the credibility of celestial phenomenon that causes the precession of equinoxes because the consequent observable retrograde movement of North Pole in circle of precession in overhead sky is absent.

\section{Orbital Spin-The Third Motion of Earth}

The celestial phenomenon observed by the astronomers of all cultures in the past indicates that:

- Vernal equinox is shifting slowly in retrograde motion annually on the equatorial plane at a rate of 50.3" seconds arc, which is termed as precession of equinoxes.

- The north pole of earth's axis is simultaneously describing a circle of precision in the same direction in overhead sky about star Polaris whose annual shift is equal to precession of equinox.

- The circle of precession of equinoxes observed on the equatorial plane is parallel to the plane of precision circle described by the North Pole in the overhead sky about star Polaris (Figure 4). When we join the point of annual shift on the above two planes which are parallel to each other over a period of 25,772 years are similar ellipses formed are one above the other.

The earlier astronomers inter-related the above two celestial phenomena and opined that this is due to either Earth's wobble or moving solar system. However, there is no logical explanation, which convinces the hypothe- 


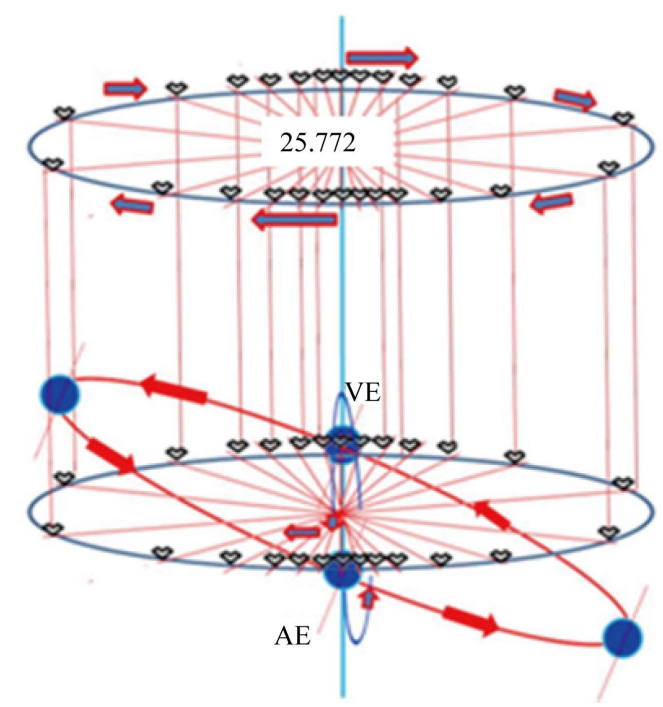

Figure 4. The third motion of Earth-Parallel circles of precession at equatorial plane and overhead sky (North Pole) described by Earth.

sis as correct and true.

Now let us examine the Moon's orbit round the Earth. Moon is the only satellite of the Earth. It is an ellipsoid. It rotates on its axis in an anti-clockwise direction that is from west to east similar to Earth's rotation on its axis. Moon's orbit round the Earth (Figure 5) is an ellipse and is inclined at 5.15 degrees to the plane of Earth's orbit round the Sun (Ecliptic) [18].

The Moon's transit around the Earth is in an anti-clockwise direction similar to the Earth's orbital motion round the Sun.

The sidereal period with reference to sars moon completes one revolution or 360 degrees in the zodiac in 27.32 solar days. Its synodic period with reference to Sun measured from New moon to next New moon day is 29.53 solar days, which is the average lunar month.

On comparison, the basic movements of Earth and Moon, are similar to each other, the orbits are elliptical the Earth's ecliptic is inclined to equatorial plane at an angle of 23.5 degrees; the Moon's orbit is inclined at 5.15 degrees to the Earth's orbital plane that is ecliptic.

The first and second motions of Moon are similar to the first and second motions of the earth. The Moon's revolution around the Earth is 29.52 days, which is similar in nature to Earth's second motion round the Sun in 365.256 days.

Earth crosses equatorial plane while orbiting round the Sun on the ecliptic at two significant points known as Vernal equinox and Autumnal equinox annually.

The annual retrograde shift of equinoxes is 50.3 seconds. When all the annual equinox points are connected, it describes an ecliptic in 25,772 years approximately as already explained. On comparison, the points of intersection that is Vernal equinox (Ascending node) and Autumnal equinox (Descending node) of earth and moon's Ascending node and Descending node are similar in existence because of identical celestial motions.

The Ascending and Descending nodes of Moon emerge during one complete revolution of Moon round the Earth. Moon's orbit intersects with the ecliptic plane of Earth at two nodal points once in 27.3 days. It transits 360 degrees covering all the signs of zodiac during one revolution.

After completion of one revolution or orbit in 27.3 days, Moon does not cross the ecliptic plane at the earlier point of intersection but re-emerges at a point that is 1 degree 26 minutes behind the earlier point (Figure 6). This rate of precision in case of Moon is to be 3 minutes 11 seconds per day approximately and in 27.3 days it is equal to 1 degree 26 minutes. Thus, retrograde motion of these nodes (nodal points) complete one revolution of 360 degrees on the ecliptic plane transiting all signs of zodiac in 6798 days or 18 years 224 days [19]. If we imagine the points of emergence when viewed from a point above the North- pole of the moon in the overhead sky it appears that the Moon's orbit is spinning in clockwise direction (Orbital spin) which is opposite to the normal 


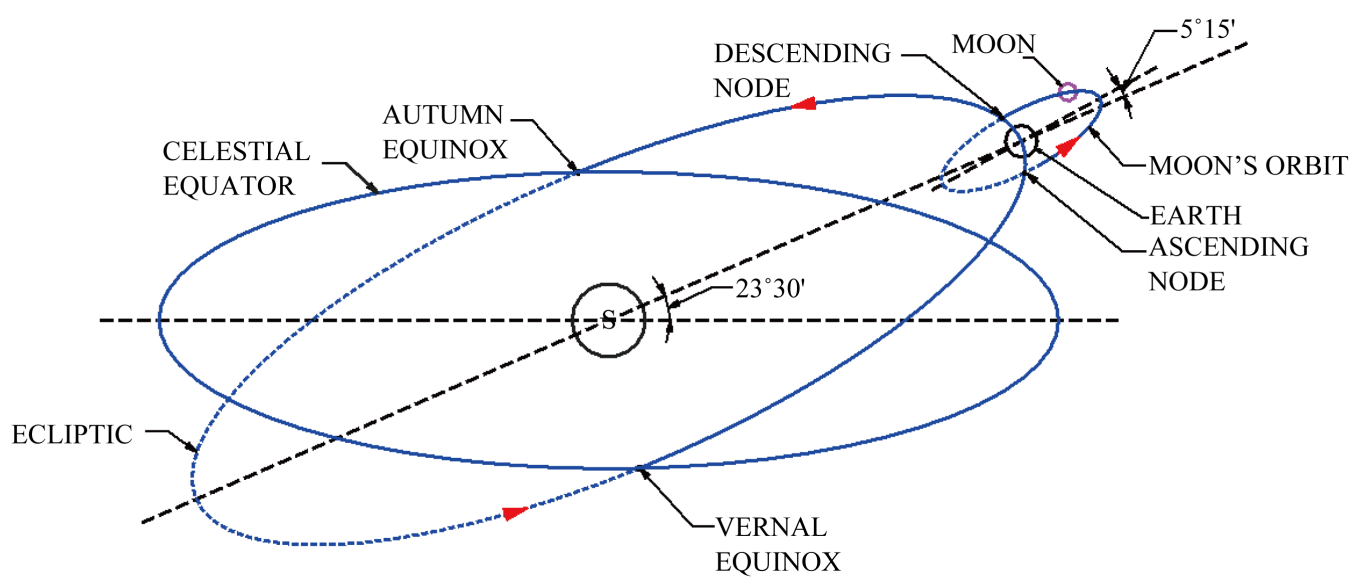

ASCENDING AND DESCENDING NODES OF EARTH AND MOON

Figure 5. Moon's orbit around Earth—Ascending and Descending nodes.

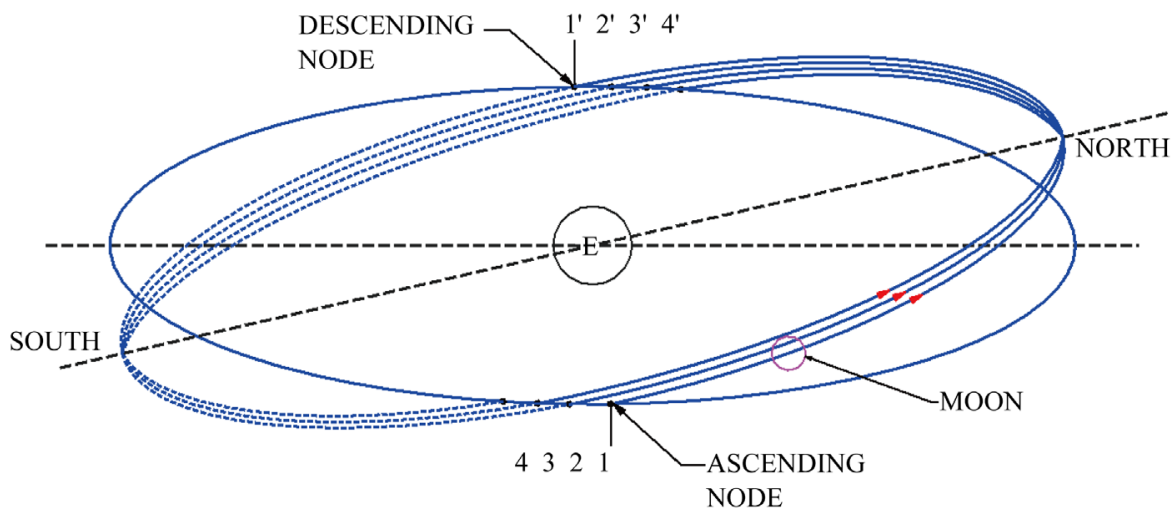

ORBITAL SPIN OF MOON

Figure 6. Orbital spin of Moon.

rotation of Moon's motion around Earth. This cosmic phenomenon precisely demonstrates that Orbital spin of Moon causes motion of Nodes on the ecliptic in opposite direction to the direction of rotation of Moon's axis. If viewed from surface of Moon, its North Pole certainly describes circle of precession in over head sky in 18.6 years roughly.

If this theory of celestial phenomena when extended to the reason of similarity in case of Earth's Vernal equinox (Ascending node) and Autumnal equinox (Descending node), it will be noticed that the retrograde motion of the precision of equinoxes is due to Orbital spin of the Earth. Earth therefore is also re-emerging on the equatorial plane at a point of 50.3 seconds behind the earlier points of equinoxes, after completing one revolution around the Sun annually. The fixed North Pole of the axis of rotation thus does not wobble but describes a circle of precession in the same direction in the overhead skies.

\section{Discussion and Conclusion}

The center of earth lies on the axis of rotation at its center. North Pole of the axis moves in a direction in which center of the axis moves. When center of the earth's axis is moving in a counter clockwise direction, any circle of precession described by North Pole in opposite direction in overhead sky is feasible unless the center of earth shifts from fixed position in opposite direction on equatorial plane on the equinox day. Such a cosmic phenomenon is plausible and feasible with the condition that while earth moving in counter clockwise direction in its orbit, it has to move in opposite direction on the equatorial plane on equinox day. It is possible when earth's or- 
bit shifts its point of apparently fixed orbit on the equatorial plane on equinox day in a clockwise direction at the rate of 50.3" arc annually opposite to its orbital direction. When we join all equinox points and view from a point above north of earth, a precession of circle on equatorial plane is obtained, which is parallel and similar to the precession of circle described by North Pole in the overhead sky.

Therefore, the precession of equinoxes i.e. the third motion of the Earth is caused due to Orbital spin of Earth (Figure 7) around the Sun when viewed from North and it is not due to Earth's wobble or due to moving solar system as surmised by the earlier astronomers.

The concept enlarges into circle of precession in respect of summer $\&$ winter solstices as well as in retrograde direction in Northern and southern hemispheres in an inclined position to the equatorial plane. The regress of summer solstice as viewed from a point above North Pole is due to the Orbital spin of Earth (Figure 8).

The annual shift of earth's orbit on equatorial plane therefore is due to orbital spin, which causes precession

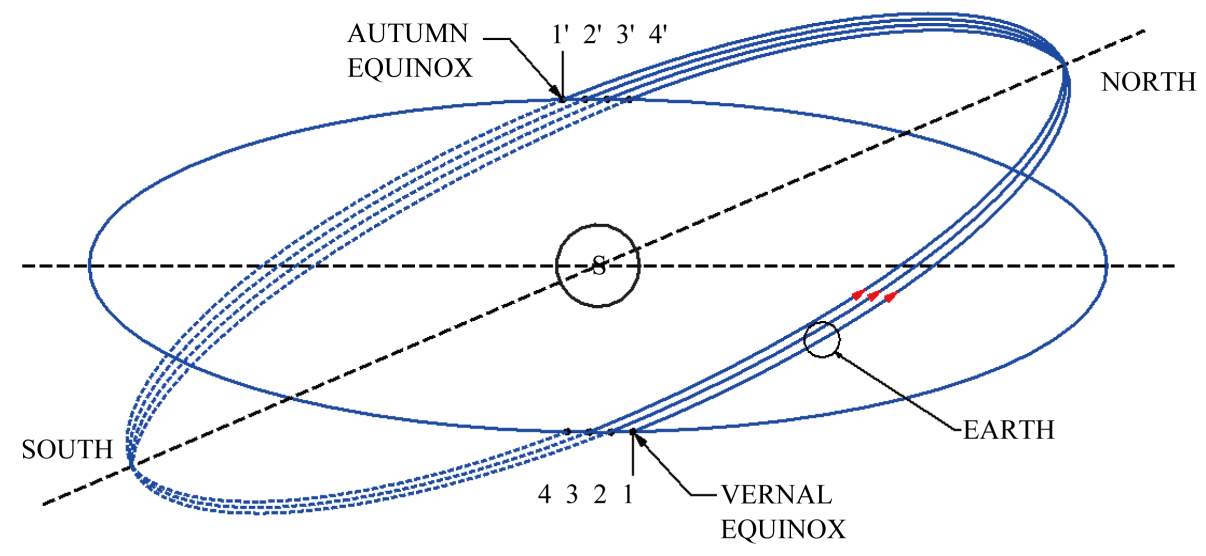

ORBITAL SPIN OF EARTH

Figure 7. The Third Motion Earth—Orbital spin of Earth.

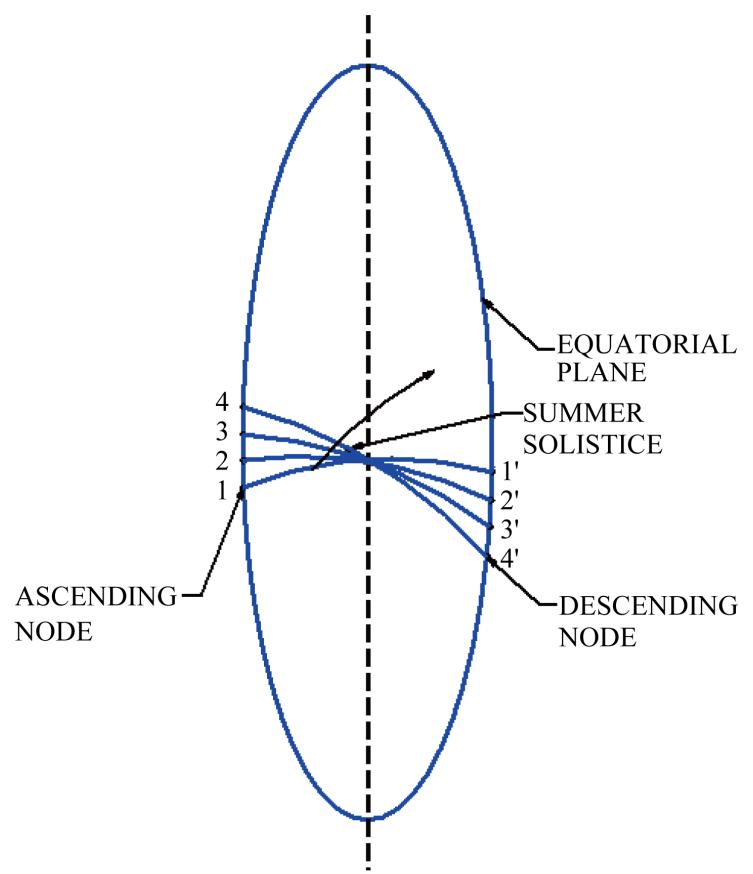

ANNUAL REGRESS OF SUMMER SOLISTICE

DUE TO ORBITAL SPIN OF EARTH

Figure 8. Regress of summer solistice-View from North. 
of equinoxes.

\section{Acknowledgements}

I thank my friends D. Venugopal—space scientist and Satyanarayana Naik—astrologer, Hyderabad, India, who rendered valuable information and suggestions during several discussions I had with them which encouraged me to write this paper and provided many refinements.

\section{References}

[1] Smart, W.M. (1986) Precession and Nutation. In: Smart, W.M., Ed., Text Book on Spherical Astronomy, 6th Edition, Cambridge University Press, Cambridge, Chapter X, 226.

[2] Wikipedia, Axial Precession, Section 2, Effects.

[3] Wikipedia, Axial Precession, Section 3.5, Middle Ages and Renaissances.

[4] Smart, W.M. (1986) Precession and Nutation. In: Smart, W.M., Ed., Text Book on Spherical Astronomy, 6th Edition, Cambridge University Press, Cambridge, Chapter X, 227-228.

[5] Wikipedia, Axial Precession, Introductory.

[6] Wikipedia, Equinox, Introductory.

[7] Wikipedia, Equinox, Section 1.2, Names

[8] Wikipedia, Equinox, Section 2, Equinox of Other Planets

[9] Wikipedia, Lunar Nodes, Section 1, Names and Symbols

[10] Wikipedia, Earth, Section 4, Orbit.

[11] Wikipedia, Tropical Year, Introductory.

[12] Wikipedia, Axial Precession, Section 6, Change in Pole Star.

[13] Wikipedia, Zodiac, Introductory.

[14] Wikipedia, Constellations, Introductory, Section 4, Asterisms.

[15] Wikipedia, Zodiac, Section 3, Introductory, 12 Signs, Astrological sign.

[16] Wikipedia, Ayanamsa.

[17] Wikipedia, Axial Precession, Section 8, Cause.

[18] Wikipedia, Moon.

[19] Wikipedia, Lunar Nodes. 